\title{
Resignificando la articulación entre investigación y la extensión en la Cátedra de Fisiología de la Facultad de Odontología (UNC), Argentina
}

\author{
Evelin Bachmeier ${ }^{1}$, Fernando Martín Wietz ${ }^{2}$, María Elena Migueles Goitea ${ }^{2}$, Jorge Alberto Linares ${ }^{3}$, \\ Claudio Gastón Dubersarsky ${ }^{2}$, Emma Gloria Ferreyra Mariani ${ }^{2}$, Ana Beatriz Finkelberg ${ }^{4}$, Marcelo
}

Adrián Mazzeo 5

Resumen: Desde 2004, la Cátedra de Fisiología de la Facultad de Odontología de la Universidad Nacional de Córdoba desarrolló actividades de investigación clínica en pacientes con diagnóstico de algunos tipos de cáncer sistémico asistidos en el Servicio de Oncohematología del Sanatorio Allende (Córdoba-Argentina). Las complicaciones bucales por quimioterapia observadas, permitieron evaluar la toxicidad de ciertas drogas oncológicas en la cavidad bucal. Un mayor conocimiento de los efectos adversos de las drogas oncológicas en la calidad de vida de dichos pacientes, motivó la realización de consultas gratuitas de diagnóstico preventivo y orientación con el propósito de minimizar tales efectos. Se brindó soporte profesional sobre cuidados bucales en estos pacientes, mediante ateneos, visitas de sala, interconsultas, promoviendo la inclusión del Odontólogo en el equipo interdisciplinario de salud. Posteriormente se firmó un convenio de cooperación mixto (Investigación / Extensión) entre la Facultad de Odontología de la Universidad Nacional de Córdoba y el Sanatorio Allende. Seguidamente se creó un Servicio de Asistencia gratuito orientado al diagnóstico y derivación de patologías bucales de pacientes sometidos a quimioterapia y Trasplante de médula ósea. En 2018 se constituyó el Primer Centro Extensionista de Orientación y Vinculación de pacientes con diagnóstico de quimioterapia ambulatoria y Trasplante de médula ósea (CEOVI). Este Centro promueve como objetivos continuar desarrollando funciones con amplio sentido social, propiciando un intercambio de experiencias y generando estrategias educativas para la formación de profesionales de la salud en el diagnóstico y manejo de patologías bucales asociadas a quimioterapia. CEOVI estimula acciones colaborativas con otras instituciones que aborden dicha temática, integrando la labor científica y clínica con la actividad extensionista.

Palavras-chave: Centro Extensionista; Pacientes Oncológicos; Manifestaciones Bucales

\section{Resignifying the link between research and extension in the Chair of Physiology of the School of Dentistry (UNC), Argentine}

Abstract: Since 2004, the Chair of Physiology of the School of Dentistry of the National University of Córdoba has carried out clinical research activities in patients diagnosed with some types of systemic cancer, assisted by the Onco-Hematology Service of the Allende Hospital (Córdoba, Argentina). The observed oral complications from chemotherapy allowed the evaluation of certain oncological drugs' toxicity in the oral cavity. Greater knowledge of the adverse effects of chemotherapy on these patients' quality of life led to free consultations for preventive diagnosis and counseling to minimize these effects. Professional support for oral care was provided to these patients through athenaeums, ward visits, interconsultations, and promoting the Dentist as a member of the interdisciplinary health team. Next, a mixed cooperation agreement (Research/Extension) between the School of Dentistry of the National University of Córdoba and the Allende Hospital was signed. Subsequently, a free Assistance Service was created to diagnose and refer oral pathologies in patients undergoing chemotherapy and bone marrow transplantation. In 2018, the First Orientation and Outreach Extension Center (CEOVI) was established for patients diagnosed with outpatient chemotherapy and Bone Marrow Transplantation. The objectives promoted by this Center are to continue developing functions with broad social awareness, fostering an exchange of experiences, and generating educational strategies for training health professionals in the diagnosis and management of chemotherapy-related oral pathologies. CEOVI encourages collaborative actions with other institutions that address this issue, integrating scientific and clinical work with extension activities.

Keywords: Extension Center; Cancer Patients; Oral Manifestations
Originales recibidos en

08 de julio de 2020

Aceptado en

24 de septiembre de 2020

1

Profesora Adjunta, Cátedra de Fisiología, Facultad de Odontología, Universidad Nacional de Córdoba (UNC) Argentina.

evelinbachmeier@unc.edu.ar 2

Profesores Asistentes, Cátedra de Fisiología, Facultad de Odontología, Universidad Nacional de Córdoba (UNC). fernando.wietz@unc.edu.ar

m.elena.migueles@unc.edu.ar claudio.dubersarsky@unc.edu. ar

gloria.ferreyra@unc.edu.ar 3 Bioquímico, Cátedra de Fisiología, Facultad de Odontología, Universidad Nacional de Córdoba (UNC) jorge.linares@unc.edu.ar 4 Ex Profesora Titular, Cátedra de Fisiología, Facultad de Odontología, Universidad Nacional de Córdoba (UNC) ana.finkelberg@unc.edu.ar 5 Profesor Titular, Cátedra de Fisiología, Facultad de Odontología, Universidad Nacional de Córdoba (UNC). marcelo.mazzeo@unc.edu.ar (autor correspondiente) 


\section{Introducción}

El Plan de Acción "Salud para todos en el año 2000" de la Organización Mundial de Salud y Organización Panamericana de Salud refiere a la Salud como un derecho social, fundamental del Ser Humano, por lo que la salud bucal no puede ni debe desprenderse de esta afirmación. En el mundo globalizado en que vivimos, la realización de estos derechos, se convierte en un objetivo prioritario de las sociedades, incluyendo también, el derecho al acceso a la salud, a servicios y sistemas sanitarios efectivos, donde la salud bucal sea una parte integral de la salud y del bienestar general. En este sentido, sería oportuno dar la posibilidad para que las profesiones sanitarias tengan una formación de calidad que permita satisfacer necesidades y demandas, fortaleciendo el compromiso social y colocando a la comunidad en el centro de la escena (World Health Organization [WHO], 1982). Por su parte, recordemos que la reforma Universitaria de 1918 acontecida en la Universidad Nacional de Córdoba tuvo un significado especial para la Educación Superior, ya que postulaba un nuevo paradigma concebido no solo como un hecho político, sino también social, integrado al aspecto educativo que se proyectaría desde Córdoba hacia toda América del Sur, el Caribe y el mundo. Este paradigma contuvo y propone en la actualidad, los principios fundamentales de un nuevo tipo de Universidad, el de la "Universidad Reformista". Dichos principios señalan aspectos relevantes como la autonomía, el desarrollo del pensamiento crítico, organización política basada en el cogobierno de todos los estamentos, libertad de organización y expresión de Cátedra con desarrollo científico y académico en términos de calidad. Estos aspectos conducirían a que la Universidad tuviera un rol protagónico y más cercano con la sociedad, contribuyendo desde el conocimiento de los problemas genuinos de la misma, a potenciar el compromiso social universitario, con el objetivo de permitir el intercambio de saberes a todos los sectores de la comunidad y a la democratización del conocimiento.

De alguna manera, este cambio de paradigma no representaba solamente un proceso de apropiación de derechos sociales, sino que implicó, además, la incorporación de las llamadas funciones sustantivas como el acceso a la salud a favor de la comunidad. Este tránsito que lleva más de cien años, estuvo atravesado por numerosas dimensiones con sus fortalezas y debilidades en el marco de nuestro sistema universitario que se repiensa constantemente para optimizar su participación en diversas tareas en territorio vinculadas al campo de la salud comunitaria (Recalde, 2008).

En este proceso, los principios vinculados a los tres pilares de nuestra Universidad Reformista no evolucionaron del mismo modo, puesto que la misión académica y científica fueron apropiándose del quehacer diario de la actividad de las Cátedras en forma más vertiginosa, dejando en cierto grado de desventaja a la tarea extensionista. El trayecto de la Cátedra de Fisiología de la Facultad de Odontología de esta cuatricentenaria Universidad no ha sido excepción de ello (Aboslaiman, 2019). Desde su constitución hasta la apropiación del sentido comunitario y su inmersión en la proposición de acciones con sentido social, debió transitar un largo recorrido para evolucionar hacia un nuevo paradigma en el que desde las ciencias básicas también se comprendiera y asimilara que era posible acompañar a la sociedad en sus múltiples necesidades (Dowbor, 2007).

\section{Metodología}

La creación del Primer Centro Extensionista de Orientación y Vinculación de pacientes con diagnóstico de quimioterapia ambulatoria y trasplante de médula ósea(CEOVI) que fue aprobado por Ordenanza 10/20218 del Honorable Consejo Directivo de la Facultad de Odontología de la Universidad Nacional de Córdoba, propone continuar desarrollando la actividad de atención odontológica gratuita en pacientes con indicación 
de quimioterapia ambulatoria y de quimioterapia de acondicionamiento en altas dosis previo al trasplante de médula ósea. Se formula la participación activa de los integrantes de la Cátedra de Fisiología y de profesionales invitados de otras Cátedras afines a estos objetivos, en colaboración con otros actores de las ciencias de la salud en forma interdisciplinaria para abordar la contención del paciente y su concientización sobre cuidados bucales previo, durante y posterior al tratamiento oncológico. Se brinda capacitación a los profesionales vinculados al Servicio de Oncohematología en el que se inició la tarea de investigación y de otras instituciones hospitalarias públicas o privadas, optimizando el intercambio de experiencias clínicas. Se impulsan estrategias educativas para la formación de profesionales de la salud en la detección y/o tratamiento de patologías bucales asociadas a quimioterapia. Las principales iniciativas tienen amplia relación con el desarrollo de acciones comunitarias entre el CEOVI y la Fundación "Todos Contra El Cáncer". Se acondicionó un espacio físico de la Cátedra para la instalación de un consultorio odontológico para recepción y diagnóstico del estado de salud de la cavidad bucal de los pacientes que recibirán o finalizarán su quimioterapia. En este consultorio se realiza historia clínica sistémica y odonto-estomatológica, previa firma de consentimiento informado. Se consigna la medicación recibida por el paciente, interpretación de estudios complementarios como radiografías y análisis clínicos y una revisación exhaustiva de la boca. A partir de ello se inicia un proceso de intercambio profesional con el Médico Oncólogo de cabecera y el Odontólogo del paciente para brindar un informe sobre las condiciones reales del sistema estomatognático y orientar el tratamiento ágil y adecuado previo a la quimioterapia. De modo tal que Paciente, Oncólogo Clínico y Odontólogo tienen conocimiento en forma simultánea del diagnóstico de situación de manera confidencial y personalizada. Desde esta perspectiva se alienta la toma de recaudos necesarios para minimizar la exacerbación de síntomas y/o enfermedades bucales durante el período de inmunosupresión parcial o completa. En caso que el paciente se viese impedido de contar con Odontólogo tratante por razones de índole económica, se efectúa derivación directa desde el Centro a los Servicios operantes de la Facultad de Odontología de esta Universidad con el objeto de dar una solución urgente y precisa a las patologías de índole bucal que podrían generar complicaciones y reducción de la calidad de vida de los mismos durante el tratamiento con drogas oncológicas. Este Centro realiza, además, actividades de seguimiento a distancia con el objeto de evaluar la evolución de las condiciones sanitarias de la boca, manteniendo un vínculo estrecho con los pacientes y su entorno. También se organizan iniciativas de difusión mediante actividades educativas respecto a la toma de conciencia social instaurando la educación sobre diversas cuestiones vinculadas a la temática.

\section{Relato de la experiencia y Discusión}

La Cátedra de Fisiología se inició como Asignatura fundacional con la creación de la Escuela de Odontología en 1915 que dependía de la Facultad de Ciencias Médicas y que continuó como tal, luego de la transformación de la Escuela en Facultad autónoma en el año 1956. Desde esta perspectiva, hacemos referencia a una trayectoria de más de cien años, forjados por la presencia de notables Profesores los cuales, desde sus albores, le dieron una impronta basada no solo en la Docencia para la formación de futuros profesionales de la Salud, sino además con una orientación que apostó a la jerarquización de la Investigación eminentemente básica con transferencia de numerosos aportes a la actividad Clínica.

Esta situación, por cierto, se mantuvo durante varias décadas en las que las principales líneas de estudio estuvieron centradas en la actividad funcional de riñón y útero. Durante fines de los años ochenta hubo un atisbo por comenzar a cambiar la orientación de las investigaciones encaminándolas hacia una línea de trabajo odontológica. En 2001, el estudio "Algunos factores que inciden en la fisiopatología de las glándulas salivales", daría paso a una nueva propuesta formulada en el año 2004, para la concreción de una Tesis Doctoral mixta que abordara por primera vez en la historia de la Cátedra, no solo las tradicionales actividades experimentales 
en animales de laboratorio, sino además la incorporación de la actividad clínica. Esta Tesis reclutaba entre sus aspectos más relevantes, el estudio de la toxicidad de las drogas oncológicas y su repercusión en la cavidad bucal en pacientes durante y posterior a la quimioterapia ambulatoria. Las actividades de investigación en esta línea nos permitieron acceder al Servicio de Oncohematología del Sanatorio Allende de la Ciudad de Córdoba con el objeto de efectuar en los primeros años, algunos estudios observacionales-longitudinales en Pacientes con diagnóstico de cáncer de colon y mama (Mazzeo, 2009).

Durante esos años conforme los resultados obtenidos, planteábamos retroalimentar a estos pacientes algunas acciones estratégicas en un intento por mejorar su calidad de vida, debido a las alteraciones más usuales ocurridas en la cavidad bucal durante el tratamiento oncológico. Estas complicaciones resultaban evidentes por su carácter no selectivo, alcanzando sistemas orgánicos como el sistema estomatognático que posee por característica distintiva un alto índice de recambio celular y una notoria actividad funcional. Una vez finalizada la Tesis en el año 2009, se inició una secuencia de hechos que fueron induciendo un proceso de interacción mayor no solo con el personal médico y de enfermería del Sanatorio referenciado, sino además un creciente intercambio dialógico de saberes con la comunidad de pacientes oncológicos y sus entornos. De este modo se abrió una nueva instancia y con ello el desafío de iniciar nuestros estudios en pacientes sometidos a trasplante de médula ósea que recibían altas dosis de quimioterapia, provocando una alteración completa de su sistema inmune, con complicaciones sustanciales en la homeostasis bucal (Bachmeier et al., 2014). De modo que nuevos integrantes de la Cátedra se sumaron a colaborar en esta instancia y con ello un incremento del compromiso institucional entre el nosocomio en el cual veníamos desempeñándonos y nuestra Facultad. A partir de este momento sentíamos no solo la necesidad de seguir profundizando nuestros estudios en pacientes con diversos diagnósticos de cáncer sino además de comenzar una nueva era en la historia de la Cátedra en la que pudiéramos darle cabida por primera vez a la actividad extensionista. Es así que, a partir de estos antecedentes, elevamos un proyecto tanto a nivel Decanal como Sanatorial para la realización de un Convenio Específico de Cooperación entre la Facultad de Odontología U.N.C. y el Sanatorio Allende de la Ciudad de Córdoba para formalizar la continuidad de nuestra intervención con fines científicos/extensionistas, que fue finalmente rubricado por la Sra. Decana de la Facultad y el Director Médico del Sanatorio Allende en fecha 24 de octubre de 2013. Con posterioridad solicitamos mediante expediente n: 62994/14, concretar la creación del "Servicio de Atención Odontológica para diagnóstico y derivación de patologías bucales de pacientes oncológicos, previo al trasplante de médula ósea y quimioterapia ambulatoria". El mismo fue aprobado por Resolución Decanal n: 416/14. Desde ese entonces a la fecha, se brinda orientación diagnóstica odontoestomatológica gratuita a los pacientes que concurren al servicio de Oncohematología del Sanatorio Allende, durante la fase de estudios pre trasplante y durante quimioterapia ambulatoria. Desde entonces, concurrieron varios Odontólogos de la Cátedra, en días y horarios específicos y organizados bajo la modalidad de turnos acordados con el área de Secretaría del Servicio. De este modo se cumplieron dos metas importantes, basadas en la aceptación e inclusión de los Odontólogos al equipo interdisciplinario de Salud Oncológico y en la prevención y orientación de los pacientes oncológicos desde el diagnóstico odontológico, previo al tratamiento específico. Habíamos de este modo consolidado algunos pasos entrelazando un permanente feedback desde la investigación para ponerla al servicio de la extensión. No obstante, todavía debían unirse algunos eslabones con el propósito de resignificar dicho proceso. La tarea en el Sanatorio ya era notoria y nuestra participación profesional había sido incluida en los protocolos médicos para acompañar desde la salud bucal a los pacientes previo, durante y post tratamiento oncológico. Pero a la vez, nos motivó incursionar en la posibilidad de dar un paso aún mayor, con la meta de poder alcanzar otras comunidades con problemáticas similares. De este modo, la Cátedra de Fisiología propuso crear el "Primer Centro Universitario Extensionista de Orientación y Vinculación de pacientes con indicación de trasplante de médula ósea y quimioterapia ambulatoria, (CEOVI)". Luego de varias entrevistas con autoridades del Consejo Directivo por medio de su Comisión de Vigilancia y Reglamento sobre este novedoso Centro, mediante Ordenanza HCD 10/2018, pudo 
finalmente concretarse nuestro mayor anhelo (Figura 1). Desde su inauguración, independientemente de las tradicionales visitas semanales al Hospital de día y aislamiento del Sanatorio Allende en el Servicio de Oncohematología, se coordinan las consultas de la comunidad de pacientes oncológicos de la ciudad de Córdoba y del interior de la Provincia de Córdoba en forma totalmente abiertas y gratuitas. CEOVI, brinda información, orientación, vinculación y asesoramiento a pacientes oncológicos. Este Centro tiene por objetivo concientizar a los pacientes con indicación de quimioterapia ambulatoria y trasplante de médula ósea sobre la necesidad mantener su cavidad bucal en correctas condiciones de higiene y salud, previo al inicio de tratamiento por cáncer sistémico. Se promueve la importancia de la toma de conocimiento y decisión comprometida por parte de estos pacientes para el abordaje del tratamiento odontológico por posibles lesiones bucales de tejidos duros y blandos que pudiesen estar activas en el momento del diagnóstico por cáncer. A partir de ello se pretenden minimizar posibles efectos secundarios originados por el tratamiento con drogas antineoplásicas en moderadas o altas dosis (Sonis \& Fey, 2002). Se pone especial énfasis en que el tratamiento con drogas oncológicas puede ocasionar una reducción parcial o total del sistema inmunitario, desencadenando lesiones a nivel sistémico y en modo particular sobre la cavidad bucal. Los pacientes asistidos son de ambos sexos, comprendidos entre 18 y 75 años de edad con indicación de trasplante de médula ósea y quimioterapia ambulatoria por cáncer sistémico. Si bien la condición socioeconómica no es excluyente para la atención, se da prioridad a los pacientes de clase media y baja, por cuanto no existen otros centros dedicados a este tipo de atención en el ámbito de la salud pública. Se receptan además consultas de numerosos pacientes provenientes del interior de la Provincia y del País tanto del ámbito público como del privado. En nuestro centro, son informados sobre las principales manifestaciones clínicas tales como ulceración de la mucosa, inflamación y sangrado de las encías, alteraciones en la cantidad y calidad de la secreción salival, cambios en la percepción del gusto y patologías de los huesos maxilares (osteonecrosis), entre otras. Estas complicaciones casi siempre desencadenan retrasos del tratamiento de base, aumento de gastos intrahospitalarios y disminución de la calidad de vida durante o posterior a la quimioterapia (Raber-Durlacher et al., 2004). Durante el curso del año 2020, a raíz de la pandemia por COVID- 19, se dispuso continuar la atención de pacientes oncológicos mediante consultas remotas. Se efectúa en primer lugar contacto telefónico por WhatsApp. Seguidamente se establece envío de radiografías y fotografías clínicas de la boca por el mismo medio. Posteriormente se llevan a cabo consultas personalizadas por Meet Google, Zoom o WhatsApp. El mismo criterio es utilizado para el seguimiento de los pacientes durante y posterior a la instancia de quimioterapia. Este Centro estimula, además, la inclusión del Profesional Odontólogo en el equipo interdisciplinario de salud oncológico, con el objeto de brindar asesoramiento y vinculación a pacientes y a profesionales para el abordaje clínico y resolución inmediata de patologías instaladas en la cavidad bucal con riesgo de exacerbación de tales manifestaciones durante el tratamiento específico. En relación a la aceptación e inclusión de Odontólogos en el equipo de salud oncológico, reportamos que en la ciudad de Buenos Aires (Argentina) la Fundación Fundaleu (entidad creada y orientada a combatir la Leucemia), ha sido pionera en propiciar estas incorporaciones en el equipo interdisciplinario de salud, pero solo con sentido asistencialista. Acciones de esta naturaleza y con los mismos criterios, son también efectuadas a través de algunos Servicios especializados en la temática en el ámbito de la medicina pública y privada de nuestro País. Por su parte, la bibliografía consultada, no mostró experiencias similares a la nuestra con la cuales contrastar los antecedentes extensionistas plasmados en el presente artículo.

Entre sus misiones más destacadas articula la interacción entre Odontólogos y pacientes, por medio de una pronta información sobre posibles enfermedades de la boca ocasionadas por tratamiento por cáncer sistémico. Además, se prevé la derivación de pacientes con los distintos Servicios operantes de la Facultad de Odontología de la UNC, que, por motivos económicos, no cuenten con Odontólogo de cabecera a los fines de concretar su correcto tratamiento. 
En otro sentido el CEOVI comenzó a trabajar estrechamente con la Fundación "Todos contra el Cáncer" para la concreción de iniciativas de interés comunitario. Desde esta nueva dimensión hemos sido incorporados a la Fundación como asesores científicos con transferencia a las acciones sociales (Figura 2). De este modo, efectuamos diversas acciones en territorio tales como la presentación de varias conferencias entre la que destacamos "Inflamación y cáncer", aprobada por Resolución HCD FO-UNC 142/2019 destinada a estudiantes, profesionales, docentes y público en general.

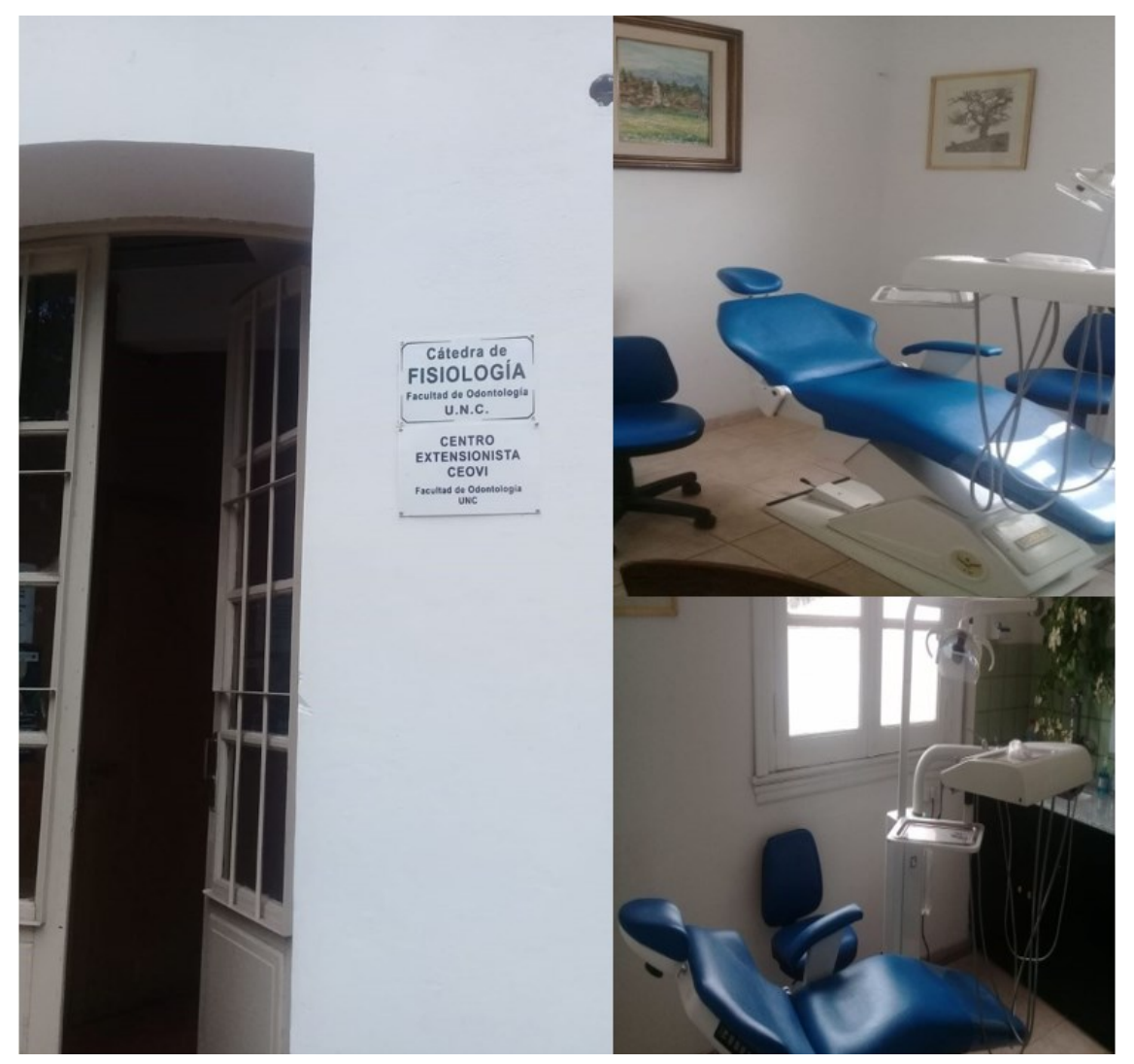

Figura 1. Ingreso a la Cátedra de Fisiología y CEOVI, Facultad de Odontología, Universidad Nacional de Córdoba (Argentina). Detalle del consultorio habilitado por Ordenanza 10/2018 para atención de pacientes oncológicos.

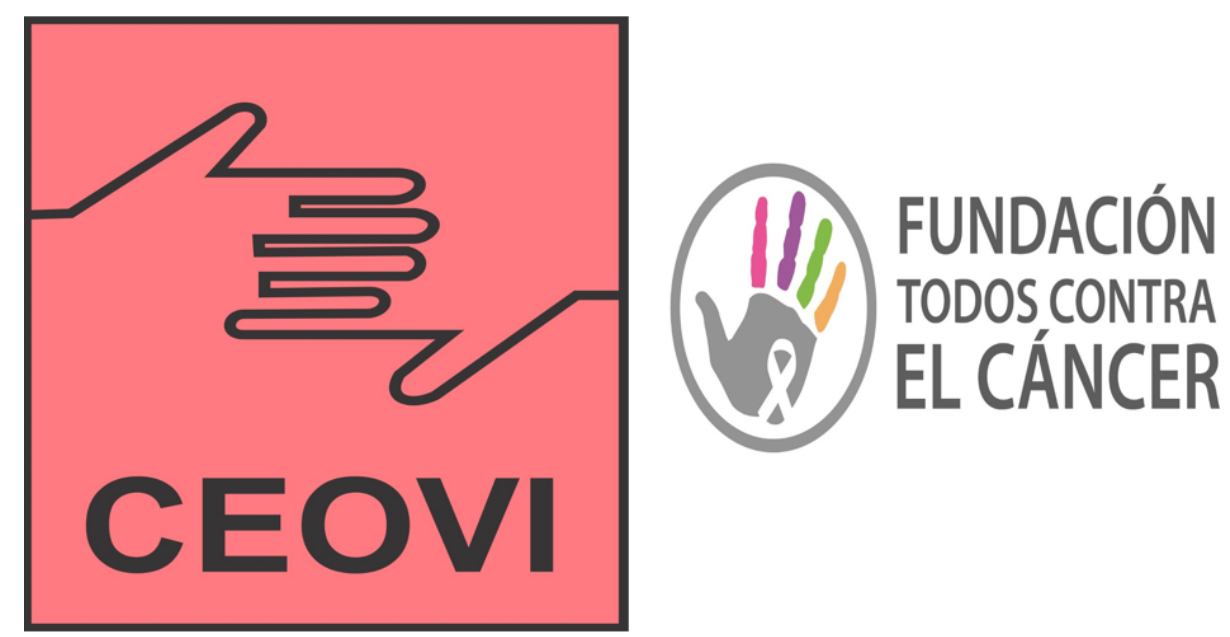

Figura 2. Logotipos que identifican las acciones en extensión del CEOVI y de la Fundación "todos contra el Cáncer". 
El CEOVI colaboró también con esta Fundación en la materialización de la Jornada "Quién cuida al que cuida", considerando la temática de contención a familiares de pacientes oncológicos.

En un esfuerzo conjunto entre ambas entidades presentamos en calidad de autores el libro titulado "Cáncer: historias de Pacientes". Se trata de una obra de divulgación destinada al entramado social, en un intento por generar desde un lenguaje coloquial, una mayor conciencia respecto de información básica sobre las diversas manifestaciones de esta enfermedad. Este libro contiene además un capítulo que narra las experiencias de algunos pacientes que fueron tratados por cáncer, con el propósito que sirva de autoayuda a otras personas con diagnósticos similares y a su entorno familiar, desde un enfoque basado en la superación de la enfermedad desde que la misma comienza a transitarse (Dubersarsky et al., 2019).

Uno de los logros más importantes de este Centro ha sido la instauración del criterio preventivo en el ámbito médico, sobre la importancia que reviste en el paciente oncológico puntualizar los tratamientos odontológicos necesarios antes de aplicar protocolos farmacológicos con drogas antineoplásicas. Solo de este modo, se podrán minimizar complicaciones funcionales transitorias tales como mucositis, disgeusia, hiposalivación, dificultades masticatorias, exacerbación de enfermedades periodontales, entre otras y permanentes, como osteonecrosis de los maxilares por uso de bifosfonatos, o hiposalivación por radiación de la zona de cabeza y cuello. El Centro cuenta con la participación estable de cuatro odontólogos, un médico oncólogo, dos bioquímicos y una médica especialista en Psiquiatría para el abordaje y contención a dichos pacientes y su entorno familiar antes, durante y post tratamiento. Los integrantes del CEOVI reflexionamos sobre la necesidad de organizar el conocimiento que se genera en extensión, vinculándolo con la docencia y la investigación (Chacin, 2008).

\section{Perspectivas para el futuro accionar comunitario del CEOVI}

Las acciones comunitarias continuarán potenciando la función social de orientación y vinculación del primer centro extensionista de la Facultad de Odontología, promoviendo intervenciones de asesoramiento a esta triada social conformada por pacientes, médicos oncólogos y odontólogos. Entre sus actividades más destacadas se tenderá a la optimización del acceso de los pacientes sin recursos, a los servicios que ofrece la Facultad de Odontología brindando de este modo una solución concreta a las principales enfermedades de la cavidad bucal de tales pacientes, previo al tratamiento específico. Proseguirá su labor docente brindando conferencias, conversatorios, cursos y talleres sobre la temática tanto para la comunidad médico-odontológica como para pacientes. Estas iniciativas conjuntas seguirán materializándose en estrecha colaboración con la Fundación "Todos Contra El Cáncer". Se integrará la participación de dos docentes de nuestra Facultad de Odontología especializados en Periodoncia, para la observación de las posibles alteraciones del tejido de soporte de los elementos dentarios en pacientes oncológicos.

Si bien el CEOVI, no contempla actualmente la participación de alumnos que cursan nuestra Asignatura, es nuestro interés que, una vez concluida la etapa de consolidación del centro extensionista, puedan efectuarse actividades con los estudiantes del grado. Fisiología ha sido estructurada en el Plan de Estudio vigente como Cátedra única ubicada en segundo año de la Carrera de Odontología, en la que cursa una cohorte anual promedio de 450 jóvenes. De modo que estarían dadas las condiciones para que en el futuro inmediato podamos invitar a los nuevos estudiantes a compartir esta experiencia por medio de conversatorios, clases teóricas abiertas y algunas acciones de promoción en territorio dentro y fuera del centro. De este modo podrían conjugarse conceptualizaciones en torno al sentido de la extensión universitaria con experiencias prácticas en la comunidad. De hecho, en un intento por vigorizar concepciones vinculadas a las nuevas praxis con el entramado social, nuestra Universidad está incursionando en la promoción de las actuales tendencias 
extensionistas a partir de numerosos proyectos consolidados en las Cátedras que funcionan en diferentes Unidades Académicas.

\section{Consideraciones finales}

Esta iniciativa posibilitó la concreción de vínculos amplios y sólidos entre los integrantes de la Cátedra de Fisiología con los actores destinatarios este particular requerimiento de la sociedad. El CEOVI concebido como un modelo innovador en las nuevas formas de llevar adelante la actividad extensionista, se ha diseñado bajo un nuevo esquema para el intercambio de saberes presentes y futuros entre la Universidad y la Comunidad.

En adhesión a los postulados de la Conferencia Regional de la UNESCO de 1996 que propició la evolución de la Educación Superior por medio de serie de paradigmas es conveniente destacar que:

"La educación en general, y la superior en particular, son instrumentos esenciales para enfrentar exitosamente los desafíos del mundo moderno y para formar ciudadanos capaces de construir una sociedad más justa y abierta, basada en la solidaridad, el respeto de los derechos humanos y el uso compartido del conocimiento y la información. La educación superior constituye, al mismo tiempo, un elemento insustituible para el desarrollo social, la producción, el crecimiento económico, el fortalecimiento de la identidad cultural, el mantenimiento de la cohesión social, la lucha contra la pobreza y la promoción de la cultura de paz." Por su parte también ligado al párrafo anterior la conferencia soslayo que: "El conocimiento es un bien social que sólo puede ser generado, transmitido, criticado y recreado, en beneficio de la sociedad, en instituciones plurales y libres, que gocen de plena autonomía y libertad académica, pero que posean una profunda conciencia de su responsabilidad y una indeclinable voluntad de servicio en la búsqueda de soluciones a las demandas, necesidades y carencias de la sociedad, a la que deben rendir cuentas como condición necesaria para el pleno ejercicio de la autonomía. La educación superior podrá cumplir tan importante misión en la medida en que se exija a sí misma la máxima calidad, para lo cual la evaluación continua y permanente es un valioso instrumento" (Organización de las Naciones Unidas para la Educación, la Ciencia y la Cultura [UNESCO], 1996).

A partir de estas convicciones, resulta más que evidente tratar de repensar sobre el valor de la investigación no como un hecho aislado en la producción de nuevos conocimientos, sino en la utilidad que debe prestar en favor de las demandas genuinas de la comunidad, como destinataria para su crecimiento.

Sabiendo que hay en el mundo otros modelos y concepciones de Universidad que obedecen a otros paradigmas como las universidades academicistas, tecnocráticas, populares o elitistas que promueven otros modelos formativos $y$, sin hacer un juicio de valor sobre el mérito de cada modelo, es importante conocer $y$ discernir al momento de optar en el ejercicio de la Docencia de Nivel Superior, en qué contexto institucional llevaremos adelante esta actividad, y si estamos debidamente identificados con dicho perfil Universitario. Desde nuestro posicionamiento sabemos que la Extensión no puede pasar inadvertida a nuestro proceso de gestión en este modelo reformista, en la permanente búsqueda para la construcción social de los conocimientos y en la apropiación e innovación de los mismos a favor de nuestra comunidad.

En este sentido, corresponde hacer referencia al reciente documento firmado por los Rectores de Universidades Hispanoamericanas sobre su propia tradición y futuro en el que, mediante una declaración firmada con motivo de los cien años de la Reforma Universitaria de Córdoba, adelantaron su opinión respecto de dichos cambios. En este documento declararon que:

"El siglo XXI avanza sin titubeos y con un dinamismo arrollador por la huella que, bien trazando el avance del conocimiento, de manera general, y la ciencia, la tecnología y las comunicaciones, de manera particular. Lo que aparece en principio como un signo auspicioso por las promesas de bienestar, también es el origen de la incertidumbre y del temor por parte de aquellos que ven 
peligrar su trabajo con el desarrollo de la automatización y la inteligencia artificial, y por los profundos interrogantes éticos que la interacción con las máquinas plantea. Asimismo, la degradación del planeta, la inseguridad alimentaria, las migraciones forzadas por catástrofes o por crisis socio-políticas, la pobreza, las dificultades en el acceso universal a la educación, la desigualad y la violencia de género, la inequidad y la violencia en todos sus matices, y el sostenido aumento de la esperanza de vida con su consiguiente impacto socio-económico, conforman problemas y desafíos globales que requieren soluciones urgentes mancomunadas inter $y$ transdiciplinarias, a la vez que sustentables. La Universidad debe constituirse en un agente de cambio que lidere las transformaciones necesarias dentro de un amplio espectro que concierne a lo tecno-científico, la normativa regulatoria, las soluciones socio-económicas, la formación ciudadana y la determinación de un horizonte ético empapado de un humanismo garante de la dignidad y los derechos humanos"(Universidad Nacional de Córdoba, 2018).

Por otra parte, y en concordancia con Gazzola (2018), es importante destacar que somos docentes de Universidades Públicas y Reformistas, donde nuestra misión "... No es solamente producir conocimientos, sino también en formar recursos humanos para el País, creando mejores condiciones para la movilidad social, para solucionar problemas, para promover la cultura y los valores humanistas (...) con sentido crítico y propositivo".

Desde nuestra participación como docentes en esta Unidad Académica y en concordancia con los principios reformistas, continuaremos promoviendo el conocimiento y su aplicación a favor de la sociedad para garantizar la democratización del saber al alcance de todos, concibiendo a la extensión como un estilo de vida con sus cúmulos de herramientas para la transformación.

La Extensión, el Voluntariado y el Servicio Comunitario Institucional, son experiencias organizadas o sostenidas por la propia Universidad, y están orientadas a promover actividades solidarias, de compromiso social y participación ciudadana por parte de los Estudiantes y Docentes, desarrollándose en paralelo con las actividades académicas. El desafío es que seamos capaces de formar Odontólogos cada vez más comprometidos con la realidad social de nuestro País, que sean capaces de vencer el aislamiento de una profesión con un fuerte componente individualista y tecnicista e integrarse con su bagaje científico a equipos Interdisciplinarios de Salud. La actividad extensionista siempre debe ser contextualizada estimulando las relaciones del conocimiento transversal del individuo en su medio y de los universitarios que llevamos nuestro conocimiento con el propósito de examinar e intercambiar experiencias para la construcción recíproca del bien común como comunidad y universidad entrelazadas en un camino de encuentros. Creemos en nuestra doble función de investigadores y extensionistas. Por ello, debemos a nuestros pacientes la oportunidad de trabajar con amplio sentido social, donde además de entusiasmo y buena voluntad, seamos capaces de poner a su disposición nuestros saberes y competencias, constancia y dedicación para poder mejorar la realidad de vida que estos y su entorno atraviesan en forma circunstancial (Veronez et al., 2019).

Creemos que la mejor forma de concluir el presente artículo que intentó narrar a grandes rasgos la historia de la creación del CEOVI y su vinculación con la comunidad es con una maravillosa reflexión de Lucarelli (2004), que plantea no solo una mirada sobre el camino transitado y sus escollos, sino sobre como evolucionaremos a partir de ello para mirar todo el trayecto que todavía nos toque recorrer:

".. son muchas las sensaciones que quedan y muchos más los aprendizajes, quedando la dulce impresión de haber evolucionado positivamente, al realizar el balance de lo andado (...) se nos corrió el velo que no nos permitía ver con claridad la dimensión de nuestro trabajo, y a pesar de las dificultades, discusiones idas y venidas, en búsqueda del camino, hemos entrado a él, se que lo estamos transitando, que quizás recién comience el recorrido, pero no de la misma manera, lo más importante de toda esta tarea transformadora, es llegar a la comprensión de que si el cambio no se produce en nuestro interior, sino cambiamos nosotros mismos nuestras actitudes, es muy 
difícil hacer cambiar a las instituciones, el mejor ejemplo está en la historia de la Cátedra observada... "(Lucarelli, 2004).

La creación del CEOVI ha permitido a una Cátedra de las Ciencias Básicas de la Facultad de Odontología de nuestra Universidad, la posibilidad de ser pionera en la construcción de otras herramientas metodológicas para la práctica extensionista integrando la investigación y su transferencia a la práctica clínica al servicio de la comunidad de pacientes oncológicos.

\section{Agradecimientos}

A las Autoridades y Equipo de Gestión del área de Biblioteca-Museo de Facultad de Odontología, a la Secretaría de Extensión Universitaria, a la Secretaría de Ciencia y Técnica (SeCyT) pertenecientes a la Universidad Nacional de Córdoba y al Servicio de Oncohematología del Sanatorio Allende de la ciudad de Córdoba. A la Odontóloga María Marcela López por su aporte científico en la creación del CEOVI y a la Lic. Tamara Cortés, Sra. Cecilia Petrini, Lic. Ana Cecilia Díaz y Lic. Carlos Facciano por su contribución en el desarrollo logístico y difusión del Centro Extensionista.

\section{Contribución de cada autor}

E. E. B., F. M. W. y E. G. F. M. actuaran en la asistencia al Servicio de Oncohematología del Sanatorio Allende y al CEOVI de la Facultad de Odontología de la Universidad Nacional de Córdoba, y participaron en la redacción del artículo y en las actividades prácticas clínicas. M. G. M. E. y J. A. L. actuaran en el procesamiento y determinaciones bioquímicas de muestras biológicas efectuadas en estudios observacionales- longitudinales sobre pacientes y en la redacción del artículo. C. G. D actuó en el asesoramiento clínico médico oncológico de los pacientes concurrentes al CEOVI de la Facultad de Odontología de la Universidad Nacional de Córdoba y en la redacción del artículo. A. B. F. dio asesoramiento científico en estudios observacionales-longitudinales sobre pacientes y participó en la orientación y corrección del artículo. M. A. M. ha ejercido la dirección de todas las iniciativas extensionistas, y fue impulsor de la creación del CEOVI de la Facultad de Odontología de la Universidad Nacional de Córdoba; participó en el planeamiento, ejecución, confección y edición en la redacción del artículo y de las actividades prácticas del proyecto.

\section{Referencias}

Aboslaiman, L. (2019). Sociedad y Universidad en la construcción de la Extensión. ExT: Revista de Extensión de la UNC, 9, 23925.

Bachmeier, E., Mazzeo, M. A., López, M. M., Linares, J. A., Jarchum, G., Wietz, F. M., \& Finkelberg, A. B. (2014). Mucositis and salivary antioxidants in patients undergoing bone marrow transplantation (BMT). Medicina Oral, Patología Oral y Cirugía Bucal, 19(5), e444.

Chacín, B. (2008). Modelo teórico-metodológico para generar conocimiento desde la extensión universitaria. Laurus, 14(26), 56-88.

Dowbor, L. (2007). Educação e apropriação da realidade local. Estudos Avançados, 21(60), 75-90.

Dubersarsky, C., Orlando, A., Mazzeo, M. A., Bachmeier, E., \& Visotsky, S. (2019). Cáncer: Historias de pacientes. Buenos Aires: Editorial Autores de Argentina.

Gazzola, A. L. En: “Cien años de desafíos: los caminos de la Universidad Pública en Latinoamérica”. Discurso pronunciado por la Ex Rectora de la Universidad Federal de Minas Gerais (UFMG) el 25.04.2018 con motivo de recibir el Doctorado Honoris Causa otorgado por la Universidad Nacional de Córdoba, 2018. Recuperado de http://www.cumbreacademica2018.unc.edu.ar/wp-content/uploads/sites/99/2018/04/Discurso-Gazzola.pdf 
Lucarelli, E. (2004). Prácticas innovadoras en la formación del docente universitario. Educação, 27(54), 503-524.

Mazzeo, M. A. Alteraciones fisiopatológicas en saliva humana y en glándulas salivales de ratas tratadas con drogas oncológicas. Phd Thesis. Universidad Nacional de Córdoba, Córdoba, 2009. Recuperado de https://rdu.unc.edu.ar/bitstream/handle/11086/5131/Mazzeo\%2C\%20Marcelo\%20Adr\%C3\%ADan\%20\%20\%28Doctor\%20en\%20Odontolog\%C3\%ADa\%29\%20Facultad\%20de\%200dontolog\%C3\%ADa.\%20Universidad\%20 Nacional\%20de\%20C\%C3\%B3rdoba\%2C\%202009.pdf?sequence=1\&isAllowed=y

Organización de las Naciones Unidas para la Educación, la Ciencia y la Cultura (1996). Declaración de la Conferencia Regional sobre Políticas y Estrategias para la Transformación de la Educación Superior en América Latina y el Caribe. Educación Superior Siglo XXI. La Habana, Cuba, UNESCO, 18 al 22 de noviembre 1996 . Recuperado de https://www.oei.es/historico/oeivirt/superior3.htm

Raber-Durlacher, J. E., Barasch, A., Peterson, D. E., Lalla, R. V., Schubert, M. M., \& Fibbe, W. E. (2004). Oral complications and management considerations in patients treated with high-dose chemotherapy. Supportive Cancer Therapy, 1(4), 219-229.

Recalde, A. (2008). Reflexiones sobre la Reforma Universitaria del año 1918: Siete hipótesis para el análisis. Question/Cuestión, 1(19): 1-12.

Sonis, S. T., \& Fey, E. G. (2002). Oral complications of cancer therapy. Cancer, 16(5), 680-686.

Universidad Nacional de Córdoba (2018). Declaración de Rectores Hispanoamericanos con motivo de los cien años de la Reforma Universitaria de Córdoba, Argentina. Córdoba: Universidad Nacional de Córdoba.

Veronez, L. C., Salomão, K. B., das Chagas, P. F., Barbieri, M. R., Scrideli, C. A., \& Tone, L. G. (2019). Genética e imunologia do câncer para alunos do ensino básico: Relato de uma experiência. Revista Brasileira de Extensão Universitária, 10(2), 63-70.

World Health Organization (1982). Health for all by the year 2000: Plan of action for the implementation of regional strategies. Washington D. C.: WHO. Recuperado de https://iris.paho.org/bitstream/handle/10665.2/39469/ 4287.pdf?sequence=1\&isAllowed $=y$

Como citar este artigo:

Bachmeier, E., Wietz, F. M., Goitea, M. E. M., Linares, J. A., Dubersarsky, C. G., Mariani, E. G. F., Finkelberg, A. B., \& Mazzeo, M. A. (2020). Resignificando la articulación entre investigación y la extensión en la Cátedra de Fisiología de la Facultad de Odontología (UNC), Argentina. Revista Brasileira de Extensão Universitária, 11(3), 377-387. https://periodicos.uffs.edu.br/index.php/RBEU/ article/view/11600/pdf 